\title{
Análisis lingüístico y estereotipos en una sentencia penal chilena*
}

\author{
Linguistic Analysis and Stereotyping in a Chilean Criminal \\ Sentence
}
Claudio Agüero San Juan** Juan Pablo Zambrano-Tiznado**, Federico Arena ${ }^{*}$, Rodrigo Coloma Correa

\begin{abstract}
RESUMEN
El artículo usa elementos de la lingüística sistémico funcional desarrollados por la escuela de Sidney junto con la teoría de estereotipos para analizar dos fragmentos de una sentencia penal chilena. La sentencia fue elegida de forma intencionada. La investigación aplicó una segmentación al texto y seleccionó dos de ellos para hacer un análisis lingüístico y de estereotipos. El análisis muestra que los jueces evaluaron la credibilidad del único testigo de la defensa de forma parcial. A su vez, el análisis de estereotipos dio cuenta de la presencia de un estereotipo normativo implícito en el texto de la sentencia, el cual se refiere al rol de madre que le cabría a la testigo en relación con el acusado. La conclusión es que el uso del estereotipo no está justificado, porque los jueces no presentaron evidencias de que el estereotipo usado fuese aplicable al caso.
\end{abstract}

Palabras clave: Análisis del discurso, sentencia penal, teoría de estereotipos, discurso jurídico, discurso ideológico.

Este artículo es resultado del proyecto Fondecyt Regular no 1190702 titulado "Lenguaje claro, palabras técnicas y conceptos jurídicos indeterminados: el razonamiento judicial sobre la ley AUGE". Este artículo también contó con el apoyo del del proyecto de investigación "Discriminación e igualdad. Un análisis filosófico de la relevancia jurídica de los estereotipos"

"* Chileno. Doctor en Ciencias Humanas mención Discurso y Cultura. Profesor de las Facultades de Derecho de las universidades Diego Portales y Alberto Hurtado, Santiago, Chile. aguero.claudio@gmail.com

*** Chileno. Doctorando en Ciencias Humanas mención Discurso y Cultura. Profesor del Departamento de Ciencias Jurídicas de la Universidad de La Frontera, Temuco, Chile. juanpablo.zambrano@ufrontera.cl

An** Argentino. Doctor Europeo en Filosofía del Derecho y Bioética Jurídica. Investigador asistente, CONICET-Universidad Nacional de Córdova, Córdoba, Argentina. fjarena@gmail.com.

Chileno. Doctor en Derecho. Profesor de la Facultad de Derecho de la Universidad Alberto Hurtado, Santiago, Chile. rcoloma@uahurtado.cl 


\begin{abstract}
This article uses elements of systemic functional linguistics developed by the Sydney School, along with stereotypes theory to analyze two fragments of a Chilean criminal sentence, which was chosen intentionally. The research applied a segmentation to the text and selected two segments to perform a linguistic and stereotypes analysis. The analysis shows that the judges partially evaluated the credibility of the only defense witness. In turn, the analysis of stereotypes shows the presence of a normative stereotype implicit in the text of the sentence. This stereotype refers to the witness' maternal role in her relationship with the defendant. It is concluded that the use of the stereotype is not justified because the judges did not present any evidence that the stereotype used was applicable to the case.
\end{abstract}

Keywords: discourse analysis, criminal sentence, theory of stereotypes, legal discourse, ideological discourse. 


\section{Introducción}

Las sentencias judiciales son textos que comunican no solo las normas aplicadas y las acciones juzgadas, sino también una serie de creencias, opiniones, elecciones y decisiones que son asumidas (explícita o implícitamente) por los jueces (Zambrano, 2015).

Las sentencias expresan la cultura jurídica chilena más allá de reflejar con fidelidad los hechos del caso, los medios de prueba y las normas aplicadas. Con estas ideas en mente, pensamos que el análisis del discurso y la teoría de estereotipos son dos buenas herramientas para lograr desentrañar qué tipo de significados están implicados en el texto de la sentencia, ya que nos permiten comprender qué tipo de juicios evaluativos comunica el texto. En este sentido, esta investigación se formula como un ejercicio que fija altos estándares de rendición de cuentas a los textos que producen los tribunales orales chilenos en lo penal. Creemos que esta aproximación es adecuada por tres razones. En primer lugar, la sentencia penal es un texto oficial del sistema de justicia criminal que tiene la potencialidad de restringir la libertad de una persona. En segundo término, la sentencia es un género discursivo especializado (Agüero, 2014; García \& Agüero, 2014) que es elaborado por un tribunal compuesto por tres jueces profesionales. Ya que la colegialidad es un mecanismo de control del proceso de decisión, parece razonable que se exija a los magistrados el máximo cuidado en la escrituración del texto que justifica la decisión judicial. Por último, las sentencias penales tienen un valor memorativo, porque están cargadas de las tensiones sociales imperantes en el grupo humano que las produce y, por ello, es sensato exigir de los jueces que usen cuidadosamente las palabras para evitar comunicar creencias, valores, posturas ideológicas o actitudes personalísimas, opuestas a las creencias sociales compartidas o directamente lesivas del estado constitucional democrático de derecho.

Para lograr nuestro objetivo usamos una sentencia dictada en 2007 por el Tercer Tribunal de Juicio Oral en lo Penal de Santiago, con el objetivo de mostrar las estrategias lingüísticas que usan los jueces para justificar las decisiones que toman y cómo estas conllevan a malos entendidos, porque las interpretaciones posibles del texto de la sentencia comportan estereotipos normativos ajenos al ideal de razonamiento judicial. 
El marco teórico-metodológico usó elementos de dos teorías de la lingüística sistémico funcional de la escuela de Sidney: la teoría del registro y el género y la teoría de la valoración. Usando este marco conceptual, constatamos la presencia de evidencias lingüísticas que permiten comprender cómo el tribunal se ha hecho cargo de la declaración de uno de los testigos del caso. Los pasos del análisis lingüístico fueron tres. En primera instancia segmentamos el texto de la sentencia siguiendo la estructura discursiva adelantada por Agüero y Zambrano $(2009,2010)$ y por Agüero (2014) para entender, desde la teoría del registro y el género (de ahora en adelante R\&GT, por su sigla en inglés), cuáles son las piezas lingüísticas de la sentencia y cómo funcionan. A cada uno de estos fragmentos los denominamos microgéneros, de los cuales nos interesan solo tres de un total de 12: aquel donde se exponen las versiones de los hechos ofrecidas por las partes; aquel en el que se hace una crónica de los medios de prueba; y aquel donde se evalúan los medios de prueba.

El segundo paso del análisis recurre a la teoría de la valoración (AT, por su sigla en inglés). Usando el sistema de actitud y, específicamente, el subsistema de juicio observamos cómo el texto explicita un estereotipo - que el tribunal considera como patrón de comportamientopara juzgar la actuación de un testigo y sus afirmaciones durante el juicio. El tercer paso del análisis consiste en usar la teoría de los estereotipos e introducir una distinción entre tipos de estereotipos que, desde nuestro punto de vista, permite identificar con mayor precisión de qué manera el patrón de conducta opera dentro del razonamiento de los jueces.

Concluimos que el análisis propuesto puede ser usado para otras sentencias judiciales, aunque las conclusiones que se presentan aquí no son generalizables.

\section{Marco de referencia}

La presente investigación se realizó bajo el alero de la lingüística sistémico funcional (de ahora en adelante SFL, por su sigla en inglés) de la escuela de Sidney, de su gramática y sus desarrollos posteriores: la R\&GT y la AT. Esta teoría lingüística desplaza el propósito social del texto desde el registro hasta el género, para configurar una organización estratificada del contexto en donde el registro es una interfaz en- 
tre el análisis del contexto social y la organización metafuncional de los recursos lingüísticos (Ciapuscio, 2005). El modelo del lenguaje de la SFL considera una triple estratificación del mismo y la recursividad de los tres estratos, esto es, afirma que el género - entendido como la función social del texto- se realiza en el registro lingüístico del texto y que este, a su vez, se instala en el lenguaje. Esta afirmación es propia de los sistémicos de Sydney y representa una de las bases de la teoría del registro y del género R\&GT (Ciapuscio, 2005).

De igual modo, la SFL define el concepto de género en términos extralingüísticos mediante la noción de función social. Esta definición disocia los aspectos semánticos de los contextuales y permite entender que los géneros son guiones culturales o formas prototípicas de construir significados que orientan las etapas de desarrollo de los textos (Ghio y Fernández, 2008). Así, el género es entendido como un proceso social o una tarea culturalmente definida (Martin, 1997), a partir de lo cual lo que distingue a un género de otro es la necesidad social que satisface cada género.

La SFL sostiene que los tres tipos de significados que construyen el lenguaje (las metafunciones ideacional, interpersonal y textual) pueden identificarse a partir de la observación de las selecciones del hablante sobre los patrones semántico-discursivos y las estructuras gramaticales en un texto. La identificación es posible porque quien observa conoce cuáles son las estructuras léxico-gramaticales que son recurrentes (obligatorias, optativas y prohibidas) dentro del registro que expresa típicamente el género. Por ello, si nos pidieran determinar qué género se expresa en preguntas como: “ ¿El policía que lo detuvo le leyó sus derechos?”, “¿Usted usará su derecho a guardar silencio o declarará en este juicio?”, o “¿Usted disparó al guardia de seguridad?”, la respuesta seguramente sería una declaración judicial. Esta respuesta es muy probable, ya que cada cultura integra de un modo específico y predecible los tres elementos del registro lingüístico: campo, tenor y modo, y esta singularidad permite a los hablantes reconocer los géneros.

El modelo de lenguaje de la SFL hasta aquí descrito permite observar el proceso de lectura como una operación de inferencia del contexto a partir del texto y el proceso de escritura como una labor de realización del contexto en un texto (Moyano, 2007). Ambos procesos 
no son ajenos a la presencia de relaciones valorativas o ideológicas y para analizar dichas relaciones la escuela de Sidney amplió el sistema de instanciación de significados interpersonales postulado por la SFL. Este desarrollo se denominó teoría de la valoración (Appraisal Theory, AT por sus siglas en inglés) y su objetivo es comprender la experiencia social mediante la constitución de roles, relaciones sociales y relaciones valorativas y/o ideológicas en los textos y en la manera en que los lectores $\mathrm{u}$ oyentes negocian esos significados (Bolívar, 2007). Para permitir el análisis de estas categorías, trabajos como los de Oteíza (2006; 2009), Hood y Martin (2005), White (2000) y Martin $(1997 ; 2000)$ han reconfigurado y profundizado en las dimensiones del tenor, construyendo un aparataje conceptual que permite determinar de qué modo y con qué fines retóricos los escritores o hablantes adoptan:

- una posición ideológica (actitudinal) hacia el significado experiencial de sus enunciados (personas, hechos, objetos, etc.);

- una postura dialógica frente a sus interlocutores reales o potenciales; y

- una perspectiva intertextual hacia la heterogeneidad de posiciones sociales, puntos de vista y/o concepciones de mundo (heteroglosia).

Cada una de estas posiciones es organizada en un sistema: actitud, gradación y compromiso.

El sistema de actitud es el que coordina las evaluaciones que realiza el hablante. Este sistema se divide en tres subsistemas: afecto, apreciación y juicio. Todos ellos pueden adquirir valores negativos, positivos o ambos a la vez (White, 2000). El subsistema de afecto se ocupa de los sentimientos ante un objeto, circunstancia o persona y en él se gestionan significados tales como los de inclinación, (in)felicidad, (in) seguridad e (in)satisfacción. El subsistema de apreciación, en tanto, atiende a la evaluación que se hace respecto de las cosas, fenómenos naturales, productos y procesos desde una perspectiva estética y no estética, como la utilidad (Oteíza, 2009). Por último, el subsistema de juicio guarda relación con la evaluación de una conducta respecto de parámetros sociales convencionales o institucionalizados. Este subsistema se divide, a su vez, en dos dimensiones: la de estima social y la de sanción social. La primera está integrada por los significados que se 
asocian con la ponderación de una conducta en referencia a un patrón sociocultural; en este caso el incumplimiento del patrón no implica una represión social coordinada, en tanto que sus valores asociados son normalidad, capacidad y tenacidad. La dimensión de la sanción social, por otro lado, está integrada por la evaluación de la veracidad que es un juicio acerca de la honestidad, en tanto que la evaluación de la adecuación constituye una valoración de la irreprochabilidad ética o moral. En ambas dimensiones, la reacción ante el incumplimiento del patrón o directiva implica una sanción más o menos institucionalizada socialmente (White, 2000).

Por último, el sistema de actitud analiza cómo se juzga el comportamiento de las personas. Aquí hemos ampliado un poco el uso de las categorías para juzgar al acto de habla del testimonio en la audiencia de juicio oral. Esta ampliación se justifica porque el juicio a la persona que declara (la testigo) está relacionado con la evaluación del contenido de su declaración.

\section{La sentencia penal como macrogénero}

El estado del arte respecto de este tema es relativamente precario en español, aunque el análisis de documentos jurídicos no es nuevo en las investigaciones lingüísticas (Campos, 2007). Las sentencias judiciales han sido estudiadas en trabajos como los de Azuelos-Atías (2006; 2007; 2010), Montolío y López (2008) y López (2006, 2010a, 2010b). No obstante, y dentro de lo que hemos investigado, no hay estudios que hayan abordado este tipo de textos con el enfoque de la LSF de la escuela de Sidney. En esta teoría lingüística la necesidad de especificar el punto de contacto entre el lenguaje y el contexto generó el desarrollo de la R\&GT (Eggins, 2004).

Un análisis preliminar de las sentencias penales como género discursivo fue el realizado por Agüero (2014) sobre la base de una serie de trabajos anteriores (Agüero y Zambrano, 2009; 2010; Zambrano y Agüero, 2009a; 2009b). La conclusión de este análisis es que la "sentencia penal chilena" es un macrogénero de acuerdo con el concepto usado por Eggins y Martin (2003), es decir, es un texto que funciona como una unidad que "contiene" una variedad de géneros. Aunque el análisis de un corpus de sentencias realizado por Agüero (2014) no es estadísticamente representativo del quehacer judicial chileno, el resultado 
de esa investigación es una descripción preliminar del género sentencia penal chilena. Otros dos estudios referidos a las sentencias penales chilenas (Agüero, 2014; Agüero y Zambrano, 2009) mostraron cuáles son los pasos en que se verifica el macrogénero. La segmentación del texto es funcional en la medida en que cada parte o microgénero es definida por una o varias funciones retóricas (Ghio y Fernández, 2008). La definición operacional de cada segmento se encuentra en Agüero y Zambrano (2009) y no profundizaremos en ella por razones de espacio, pero sí conceptualizamos con profundidad los segmentos que cuentan las acciones juzgadas, los dichos de los testigos y los medios de prueba. La elección de los segmentos está determinada por su obligatoriedad a la tarea que cumplen como microgéneros y a la fuerza que tienen dentro del conjunto. Así, los segmentos donde se narran los hechos y se valoran las pruebas como si fuesen razones para decidir el caso son, probablemente, dos de los microgéneros que más contribuyen al logro de la función social que cumple el macrogénero: justificar una decisión institucional.

\subsection{Tres segmentos de la sentencia}

Los segmentos "exposición de los hechos", "medios de prueba" y "valoración de los medios de prueba" son tres partes de un total de 12. El primero narra y describe lo que los abogados dijeron que ocurrió. Así se cuentan los hechos que son juzgados. El segundo narra y describe lo que dijeron los testigos, los peritos y la interpretación que dieron los abogados a las pruebas materiales como fotografías, armas o videos, entre otros medios de prueba. El tercero, argumenta, narra y describe las razones que tomaron en cuenta los jueces para dar valor a los medios de prueba presentados por los abogados.

Los segmentos exposición de los hechos y medios de prueba cumplen importantes funciones narrativas y descriptivas, ya que son verdaderas crónicas insertas dentro de una argumentación. El primero se concentra en la crónica del conflicto ocurrido, mientras que el segundo, lo hace en las pruebas que presentaron las partes. En cambio, el segmento valoración de los medios de prueba es típicamente argumentativo, porque su función principal es justificar el valor que da el juez a cada evidencia y solo narra y describe de forma vicaria. 
En una sentencia penal las ideologías se manifiestan en el conjunto de elecciones lingüísticas que configuran el registro (Halliday \& Hasan, 1985; Halliday, 1978; Martin \& Rose, 2003). Sin embargo, en los tres segmentos individualizados se encuentran las mejores evidencias lingüísticas, narrativas y semióticas acerca de cómo los jueces comunican sus reflexiones de los hechos del caso. Esto es así porque estos segmentos contribuyen de modo importante al logro de la función social que cumple el género mediante la certificación de los hechos. Estos segmentos responden tres preguntas: ¿Qué pasó? ¿Qué hicieron los que participaron de esas acciones? y ¿Qué justifica esas acciones?

\section{El caso y su análisis}

El caso RIT 28-2007 fue resuelto por una sala del Tercer Tribunal Oral en lo Penal de Santiago. El acusado, a quien llamaremos Andrés ${ }^{1}$, fue condenado por unanimidad a la pena de cinco años y un día como autor del delito de robo con violencia ${ }^{2}$.

La sentencia fue seleccionada de modo intencionado, porque el razonamiento probatorio se focalizó en la verosimilitud de las declaraciones de la víctima, del acusado y de la madre de este. Buscamos un caso sin peritos, porque nos pareció que esa prueba aumentaba la posibilidad de que el juez aceptara sin más sus afirmaciones al estar revestidas de un halo de cientificidad. Entonces, si los peritos poseen una cuota de credibilidad que no muestran las declaraciones de los testigos, seleccionar un caso que no presenta pruebas periciales asegura la no interferencia de este tipo de discursos en el razonamiento judicial.

\subsection{La jerarquía de las voces de la sentencia}

La distinción entre el sujeto empírico y locutor es útil para diferenciar al juez que escribe la sentencia de los responsables de los enunciados.

\footnotetext{
Hemos cambiado los nombres de las víctimas, del acusado y de los testigos con el fin de resguardar su identidad.

2 Un dato importante a tener en cuenta es que los únicos medios de prueba relevantes que se presentaron para sustentar la acusación fueron declaraciones: la de la trabajadora de casa particular que se encontraba en el lugar, la de la dueña de la residencia, la de la hija de esta última y la de un funcionario policial. La Defensoría Penal Pública presentó las declaraciones del acusado y las de tres testigos, uno de los cuales era la madre del acusado.
} 
El juez escribe y recoge voces del Ministerio Público, de la defensa, del acusado, de la víctima y de los testigos. En relación con estos locutores, el tribunal se constituye en otra voz que oficia como el único intermediario entre todas las otras voces, porque presenta cada uno de sus puntos de vista y el suyo propio. En este sentido, la actitud del juez como locutor es una parte central de la instanciación lingüística del razonamiento judicial.

Esta actitud, en el sentido de Ducrot (1988), está mediada por la que tiene el locutor en relación con los enunciadores (Ducrot, 1983). Entonces, el modo en que el juez-locutor se relaciona con los otros intervinientes-locutores se expresa a través de la evaluación de sus enunciaciones. Las ideas de Ducrot han sido desarrolladas por la escuela escandinava de la polifonía (ScaPoLine), la que señala que los enunciados de actitud expresan un punto de vista que corresponde a una entidad semántica compuesta por un origen, un juicio y un contenido. El punto de vista toma, entonces, esta forma:

$$
[\mathrm{x}](\text { juzga }(\mathrm{p}))
$$

En esta formalización, "x" simboliza el origen, es decir, el enunciador; "juzga" corresponde a la emisión de un juicio y "p" es un contenido proposicional (Nølke, Fløttum \& Norén, 2004). En la sentencia que analizamos, por ejemplo, el juez locutor reconstruye un discurso del Ministerio Público (discurso referido) diciendo: "la única persona que lo sitúa en un lugar distinto es la madre [del acusado] pero su relato es poco creíble, motivo por el que el Ministerio Público pide la condena a las penas contenidas en la acusación”. El enunciado del juez es, entonces, un enunciado de tercera jerarquía que toma la siguiente forma:

$$
\left[\mathrm{x}^{3}\right]\left(\text { cierto }\left(\left[\mathrm{x}^{2}\right]\left(\text { juzga }\left(\left[\mathrm{x}^{1}\right] \text { (juzga }(\mathrm{p})\right)\right)\right)\right)
$$

El diagrama representa la situación de las muñecas rusas llamadas matrioshka, donde la muñeca de mayor tamaño alberga en su interior a una más pequeña. Donde $\mathrm{x}^{3}$ es el punto de vista del juez-locutor; $\mathrm{x} 2$ el punto de vista del Ministerio Público-locutor como interviniente; y xl el punto de vista de la testigo-locutor (que es la madre del acusado). 
De este modo, queda bastante claro que el juez-locutor se relaciona con los intervinientes a través de sus enunciaciones.

\subsection{Análisis lingüístico}

Analizamos la instanciación lingüística de la evaluación que hacen los jueces de la declaración de la madre del acusado. Tal declaración, en el evento de que hubiera sido creída por los jueces, imponía mayores cargas de argumentación al Ministerio Público para derrotar la presunción de inocencia que favorece al acusado ${ }^{3}$. La composición del texto de la sentencia se muestra en la Tabla 1.

Tabla 1.

Composición del texto de la sentencia.

\begin{tabular}{|c|c|c|c|}
\hline $\begin{array}{l}\text { Considerandos } \\
\text { y párrafos del } \\
\text { texto de la } \\
\text { sentencia }\end{array}$ & Segmento & $\begin{array}{l}\text { Modo de } \\
\text { organización } \\
\text { del discurso } \\
\text { preferente }\end{array}$ & Fragmentos analizados \\
\hline $\begin{array}{l}\text { Considerandos } \\
2^{\circ}, 7^{\circ}, 8^{\circ}\end{array}$ & $\begin{array}{l}\text { Exposición } \\
\text { de los } \\
\text { hechos }\end{array}$ & $\begin{array}{l}\text { Narrativo y } \\
\text { descriptivo }\end{array}$ & $\begin{array}{l}\text { "Encuanto a la prueba dela defensa, } \\
\text { referente a la credibilidad de la } \\
\text { misma, primero la declaración de } \\
\text { la madre del acusado, obviamente } \\
\text { tiene interés y cariño por su hijo, sin } \\
\text { embargo, si ella tuvo conocimiento } \\
\text { que el día de los hechos su hijo } \\
\text { estuvo con ella en otra ciudad, } \\
\text { debió decirlo antes al [sic] Fiscalía o } \\
\text { al defensor, debió "poner el grito en } \\
\text { el cielo", esperó que su hijo estuviera } \\
\text { tres meses en prisión preventiva } \\
\text { sin realizar ninguna diligencia, } \\
\text { esta conducta no resulta lógica. Lo } \\
\text { mismo respecto de la declaración } \\
\text { del abogado Mario Vargas, quien } \\
\text { incluso señaló que tenía una } \\
\text { relación especial con el imputado, } \\
\text { erauno delos "regalones", silo vio en } \\
\text { la audiencia con el pelo rojo debió } \\
\text { informar de esta característica para } \\
\text { solicitar el sobreseimiento de la } \\
\text { causa, ahorrando el despliegue de } \\
\text { recursos del Estado (fragmento del } \\
\text { párrafo } 12 \text { del considerando 7o)". }\end{array}$ \\
\hline
\end{tabular}

3 En efecto, como se trataba de una testigo directa, no admite que el tribunal permanezca indiferente ante ella, a menos que muestre que es de dudosa calidad epistémica. 


\begin{tabular}{lll}
\hline Considerando & Valoración & Argumentativo, \\
$12^{\circ}$ & de los & narrativo y \\
Considerando & medios de & descriptivo \\
$13^{\circ}$ & prueba &
\end{tabular}

"Según se desprende de la
declaración de la testigo Basic
Jaque, esta sitúa al acusado, en
el lapso de tiempo en que se
cometió el ilícito motivo de este juicio, en el domicilio común de ambos ubicado en San Francisco de Mostazal, resulta así, ser la única testigo de tal circunstancia, testimonio este que se desestimará en esta parte, teniendo para ello presente la calidad de madre del acusado, lo que hace aparecer dicha declaración carente de la imparcialidad necesaria como para lograr que el tribunal adquiera más allá de toda duda razonable la convicción necesaria para una decisión absolutoria en cuanto a la participación que le cupo en el referido ilícito, ya que no es posible asegurar que no hayan existido motivaciones subjetivas que pudieran haber enturbiado su testimonio en el sentido de beneficiar a su hijo, lo que no es objetivamente posible ni razonable reprochar a una madre en las circunstancias en que aquellas pudieron producirse, considerando además la falta de precisión en cuanto a la hora de llegada del hijo al San Francisco de Mostazal, ya que mencionó, como se dijo, que fue alrededor de las 18:30 a 19:00 horas, cálculo que hizo, según lo afirmó en la audiencia, en base a que ella trabaja hasta las 18:00 horas - no refiere haber mirado el reloj- y que cuando llegó a su domicilio este ya estaba en la casa, de suyo, según los principios de la lógica y las máximas de la experiencia, no es de normal ocurrencia irse del lugar de trabajo, todos los días, exactamente a la misma hora permitida y establecida como de salida".

Fuente: Elaboración propia. 
Al revisar el texto del segmento 6 (considerando $7^{\circ}$ ) y a partir del subsistema de juicio hemos inferido la voz usada por los jueces. Luego, dividimos el texto en cláusulas y elaboramos una tabla para apreciar el análisis de transitividad y de AT de forma paralela ${ }^{4}$.

Tabla 2.

Análisis de transitividad y de AT paralelamente.

\begin{tabular}{|c|c|c|c|c|}
\hline Complejo clausal & $\begin{array}{c}\text { No de } \\
\text { cláusula }\end{array}$ & Cláusulas & Transitividad & AT \\
\hline \multirow{3}{*}{$\begin{array}{l}\text { "obviamente tiene } \\
\text { interés y cariño } \\
\text { por su hijo, sin } \\
\text { embargo, si ella tuvo } \\
\text { conocimiento, que el } \\
\text { día de los hechos su } \\
\text { hijo estuvo con ella } \\
\text { en otra ciudad, }\end{array}$} & 1 & $\begin{array}{l}\text { obviamente } \\
\text { tiene interés y } \\
\text { cariño por su } \\
\text { hijo, }\end{array}$ & $\begin{array}{l}\text { Proceso } \\
\text { relacional }\end{array}$ & $\begin{array}{l}\text { Juicio + } \\
\text { estima social } \\
\text { +tenacidad }\end{array}$ \\
\hline & 2 & $\begin{array}{l}\text { sin embargo, } \\
\text { si ella tuvo } \\
\text { conocimiento, }\end{array}$ & $\begin{array}{l}\text { Proceso } \\
\text { relacional }\end{array}$ & $\begin{array}{l}\text { Juicio + } \\
\text { estima social } \\
\text { +capacidad }\end{array}$ \\
\hline & 3 & $\begin{array}{l}\text { que el día de } \\
\text { los hechos su } \\
\text { hijo estuvo con } \\
\text { ella en otra } \\
\text { ciudad }\end{array}$ & No analizado & No analizado \\
\hline \multirow{3}{*}{$\begin{array}{l}\text { debió decirlo antes } \\
\text { al [sic] Fiscalía o } \\
\text { al defensor, debió } \\
\text { "poner el grito en el } \\
\text { cielo", }\end{array}$} & 4 & $\begin{array}{l}\text { debió decirlo } \\
\text { antes al [sic] } \\
\text { Fiscalía o al } \\
\text { defensor, }\end{array}$ & Proceso verbal & \multirow{5}{*}{$\begin{array}{l}\text { Juicio - } \\
\text { estima social } \\
\text {-normalidad }\end{array}$} \\
\hline & 5 & debió & Proceso verbal & \\
\hline & 6 & $\begin{array}{l}\text { "poner el grito } \\
\text { en el cielo" }\end{array}$ & $\begin{array}{l}\text { Nominalización } \\
\text { o metáfora } \\
\text { gramatical }\end{array}$ & \\
\hline \multirow{3}{*}{$\begin{array}{l}\text { esperó que su hijo } \\
\text { estuviera tres meses } \\
\text { en prisión preventiva } \\
\text { sin realizar ninguna } \\
\text { diligencia, esta } \\
\text { conducta no resulta } \\
\text { lógica". }\end{array}$} & 7 & $\begin{array}{l}\text { esperó que su } \\
\text { hijo estuviera } \\
\text { tres meses } \\
\text { en prisión } \\
\text { preventiva }\end{array}$ & $\begin{array}{l}\text { Proceso de } \\
\text { comportamiento }\end{array}$ & \\
\hline & 8 & $\begin{array}{l}\text { sin realizar } \\
\text { ninguna } \\
\text { diligencia }\end{array}$ & $\begin{array}{l}\text { Proceso de } \\
\text { comportamiento }\end{array}$ & \\
\hline & 9 & $\begin{array}{l}\text { esta conducta } \\
\text { no resulta } \\
\text { lógica". }\end{array}$ & $\begin{array}{l}\text { Metáfora } \\
\text { gramatical }\end{array}$ & $\begin{array}{l}\text { Juicio - sanción } \\
\text { social - } \\
\text { adecuación } \\
\text { social }\end{array}$ \\
\hline
\end{tabular}

$4 \quad$ Como se sabe, la transitividad es la red de significados que usan los hablantes para organizar su experiencia del mundo (Gil y García, 2010). Así, aunque la transitividad no es el foco del estudio, pensamos que tenerla a la vista permitirá al lector reconstruir de forma más clara el proceso de análisis, ya que muchas de las apreciaciones que hacen los jueces tiene por objeto el comportamiento de un testigo en la audiencia de juicio. 


\begin{tabular}{|c|c|c|c|c|}
\hline $\begin{array}{l}\text { Tercer párrafo } \\
\text { Por otra parte, } \\
\text { ambos testigos que } \\
\text { sitúan al imputado } \\
\text { en Santiago, lo } \\
\text { dejaron de ver a } \\
\text { las 14:00 horas y el } \\
\text { delito fue a las 20:30, } \\
\text { la única persona } \\
\text { que lo sitúa en un } \\
\text { lugar distinto es la } \\
\text { madre pero su relato } \\
\text { es poco creíble, } \\
\text { motivo por el que el } \\
\text { Ministerio Público } \\
\text { pide la condena a las } \\
\text { penas contenidas en } \\
\text { la acusación. }\end{array}$ & 10 & $\begin{array}{l}\text { pero su relato } \\
\text { es poco creíble }\end{array}$ & $\begin{array}{l}\text { Nominalización } \\
\text { del proceso de } \\
\text { relatar }\end{array}$ & $\begin{array}{l}\text { Juicio - } \\
\text { sanción social } \\
\text { adecuación } \\
\text { social (falta de } \\
\text { veracidad) }\end{array}$ \\
\hline $\begin{array}{l}\text { Solo se presentan la } \\
\text { de la madre en dos } \\
\text { cielo") y dos proces }\end{array}$ & & $\begin{array}{l}\text { aalizadas. Las clá } \\
\text { ales (debió decir } \\
\text { tamiento. }\end{array}$ & $\begin{array}{l}\text { ulas 4, } 5 \text { y } 6 \text { in } \\
\text { ' 'debió' "pone }\end{array}$ & $\begin{array}{l}\text { cian la omisión } \\
\text { grito en el }\end{array}$ \\
\hline
\end{tabular}

Fuente: Elaboración propia.

El primer párrafo reconstruye la impresión del Ministerio Público acerca de las declaraciones de tres testigos: la madre del acusado, el abogado Mariano y la testigo Rosa. Un primer comentario que merece el texto es el uso del adverbio "obviamente". La expresión originada a partir del adjetivo "obvio" permite modalizar tal calificativo, indicando que se valora una cualidad epistémica vinculada con una creencia que es aceptada por el enunciador. Así, el adverbio no solo modifica al verbo que le sucede, sino que también modaliza el enunciado y la enunciación. El uso de este adverbio es propio de un registro formal y significa que lo que se dice es evidente y que, entonces, no debe ser probado. Desde el punto de vista argumental, el adverbio refuerza la argumentación y mejora el énfasis de la aserción (Portolés, 2007). Esto puede tener una doble interpretación: una realización lingüística impropia del significado de los adverbios "verdaderamente", "naturalmente" o "realmente" y/o como una estrategia para anticipar la crítica del argumento e impedir que se desencadenen conclusiones no deseadas, pero previstas por quien argumenta ${ }^{5}$.

\footnotetext{
$5 \quad$ En este sentido, "obviamente" equivale a la expresión "de hecho" y presenta al argumento como cierto o probable.
} 
El texto reitera en tres ocasiones (dos dirigidas a la conducta de la madre) el verbo modal "deber". El uso de "debió" en el sistema de modo de la SFL puede entenderse como una modulación de obligación y de probabilidad que separa la conducta efectiva y la deseable (desde la perspectiva de los jueces). Esta lectura es plausible, ya que el verbo "deber" es usado en un modo irrealis para expresar la probabilidad de hechos pasados que no acontecieron mediante la perífrasis verbal "deber (pretérito) + infinitivo". El irrealis conduce al lector a preguntarse: ¿Qué debía haber hecho la madre? El texto responde a esta pregunta con dos conductas: "debió decirlo antes al [sic] Fiscalía o al defensor, debió 'poner el grito en el cielo'”. Más allá del desliz en la redacción, el texto expresa cómo los jueces valoran la conducta de la madre mediante un razonamiento hipotético y contrafáctico que da cuenta de que el juez escritor lo estima como posible y como obligatorio, pero que en los hechos no aconteció. Esto es así porque solo tiene sentido decir que la madre "debió" (hacer algo) en la medida en que quien enuncia la existencia del deber cree que ella estaba obligada a hacer ese algo. Ante la interrogante ¿Qué hizo la madre? el texto responde: "esperó que su hijo estuviera tres meses en prisión preventiva sin realizar ninguna diligencia”. Sobre estas conductas, ¿cuál es la opinión del tribunal? "esta conducta no resulta lógica”. Entonces, ¿cuál es la consecuencia de esta carencia en el desempeño materno? La respuesta se encuentra en el segundo párrafo: "su relato es poco creíble".

Un aspecto de interés es la cláusula "poner el grito en el cielo", expresión popular, coloquial e informal que significa la acción de protestar, llamar la atención o quejarse con vehemencia por la injusticia de una situación. El uso de la expresión es peculiar en la medida en que se trata de un registro poco ajustado a las exigencias de formalidad del lenguaje en que se escriben las sentencias. Con dicha expresión los jueces estarían dirigiéndose a un auditorio más universal, pues cualquier persona aceptaría que las madres tienen el deber de protestar de forma oportuna si a su hijo se le está acusando injustamente. Por cierto, Andrés tendría una coartada si al momento en que se cometió el delito, a su madre le hubiera constado que él estaba en otra parte. Sin embargo, la madre no sabía dónde estaba su hijo y, además, "no puso el grito en el cielo", pues solo reaccionó en la audiencia de juicio 
oral, la que fue realizada bastante tiempo después de que este había sido formalizado.

A juicio del Ministerio Público y del tribunal cualquiera que se vea afectado por la acción injusta de un tercero "debería poner el grito en el cielo". Con esta expresión los jueces quieren comunicar dos posibles conclusiones; (i) la madre inventó el relato según el cual ella estaba con su hijo en San Francisco de Mostazal y, (ii) la madre efectivamente estuvo con su hijo y no entregó oportunamente dicha información, lo que sería propio de una de una mala madre.

La Tabla 3 permite apreciar cómo los juicios acerca de la madre del acusado (ser la única testigo y tener motivaciones subjetivas) se relacionan con la evaluación de lo dicho por ella. En este sentido, es claro que la veracidad del testimonio y la credibilidad de quien declara son evaluaciones interdependientes.

Tabla 3.

Juicios acerca de la madre del acusado y su evaluación.

\begin{tabular}{|c|c|c|c|c|}
\hline Complejo clausal & $\begin{array}{c}\text { No de } \\
\text { cláusula }\end{array}$ & Cláusula & Transitividad & AT \\
\hline \multirow{4}{*}{$\begin{array}{l}\text { Según se desprende de la } \\
\text { declaración de la testigo } \\
\text { Pérez Soto, esta sitúa al } \\
\text { acusado, en el lapso de } \\
\text { tiempo en que se come- } \\
\text { tió el ilícito motivo de } \\
\text { este juicio, en el domici- } \\
\text { lio común de ambos ubi- } \\
\text { cado en San Francisco de } \\
\text { Mostazal, resulta así, ser } \\
\text { la única testigo de tal cir- } \\
\text { cunstancia, testimonio } \\
\text { este que se desestimará } \\
\text { en esta parte, teniendo } \\
\text { para ello presente la cali- } \\
\text { dad de madre del acusa- } \\
\text { do, lo que hace aparecer } \\
\text { dicha declaración caren- } \\
\text { te de la imparcialidad ne- } \\
\text { cesaria como para lograr } \\
\text { que el tribunal adquiera } \\
\text { más allá de toda duda } \\
\text { razonable la convicción } \\
\text { necesaria para una de- } \\
\text { cisión absolutoria en } \\
\text { cuanto a la participación } \\
\text { que le cupo en el referido }\end{array}$} & $\begin{array}{l}\text { No } \\
\text { analizado }\end{array}$ & No analizado & No analizado & $\begin{array}{l}\text { No } \\
\text { analizado }\end{array}$ \\
\hline & 1 & $\begin{array}{l}\text { resulta así, } \\
\text { ser la única } \\
\text { testigo de tal } \\
\text { circunstancia, }\end{array}$ & $\begin{array}{l}\text { Proceso } \\
\text { existencial }\end{array}$ & \multirow[t]{2}{*}{$\begin{array}{l}\text { Juicio } \\
\text { - estima } \\
\text { social } \\
\text { - } \\
\text { normalidad }\end{array}$} \\
\hline & 2 & $\begin{array}{l}\text { testimonio } \\
\text { este que se } \\
\text { desestimará } \\
\text { en esta parte }\end{array}$ & $\begin{array}{l}\text { Proceso } \\
\text { mental }\end{array}$ & \\
\hline & 3 & $\begin{array}{l}\text { teniendo para } \\
\text { ello presente } \\
\text { la calidad de } \\
\text { madre del } \\
\text { acusado }\end{array}$ & $\begin{array}{l}\text { Proceso } \\
\text { mental }\end{array}$ & $\begin{array}{l}\text { Juicio } \\
\text { - estima } \\
\text { social } \\
- \\
\text { normalidad }\end{array}$ \\
\hline
\end{tabular}




\begin{tabular}{|c|c|c|c|c|}
\hline $\begin{array}{l}\text { "ilícito, ya que no es po- } \\
\text { sible asegurar que no } \\
\text { hayan existido motiva- } \\
\text { ciones subjetivas que } \\
\text { pudieran haber entur- } \\
\text { biado su testimonio en el } \\
\text { sentido de beneficiar a su }\end{array}$ & 4 & $\begin{array}{l}\text { lo que hace } \\
\text { aparecer } \\
\text { dicha } \\
\text { declaración } \\
\text { carente de la } \\
\text { imparcialidad } \\
\text { necesaria }\end{array}$ & $\begin{array}{l}\text { Proceso } \\
\text { relacional }\end{array}$ & \multirow[t]{2}{*}{$\begin{array}{l}\text { Juicio } \\
\text { - estima } \\
\text { social } \\
\text { - tenacidad }\end{array}$} \\
\hline $\begin{array}{l}\text { hijo, lo que no es objeti- } \\
\text { vamente posible ni razo- } \\
\text { nable reprochar a una } \\
\text { madre en las circunstan- } \\
\text { cias en que aquellas pu- } \\
\text { dieron producirse, consi- } \\
\text { derando además la falta } \\
\text { de precisión en cuanto } \\
\text { a la hora de llegada del } \\
\text { hijo a San Francisco de } \\
\text { Mostazal, ya que men- } \\
\text { cionó, como se dijo, que } \\
\text { fue alrededor de las } 18: 30 \\
\text { a 19:00 horas, cálculo que } \\
\text { hizo, según lo afirmó en } \\
\text { la audiencia, en base a }\end{array}$ & 5 & $\begin{array}{l}\text { como para } \\
\text { lograr que } \\
\text { el tribunal } \\
\text { adquiera } \\
\text { más allá de } \\
\text { toda duda } \\
\text { razonable la } \\
\text { convicción } \\
\text { necesaria para } \\
\text { una decisión } \\
\text { absolutoria } \\
\text { en cuanto a la } \\
\text { participación } \\
\text { que le cupo } \\
\text { en el referido } \\
\text { ilícito }\end{array}$ & $\begin{array}{l}\text { Proceso } \\
\text { mental }\end{array}$ & \\
\hline \multirow{3}{*}{$\begin{array}{l}\text { 18:00 horas - no refiere } \\
\text { haber mirado el reloj- } \\
\text { y que cuando llegó a su } \\
\text { domicilio este ya estaba } \\
\text { en la casa, de suyo, según } \\
\text { los principios de la lógica } \\
\text { y las máximas de la expe- } \\
\text { riencia, no es de normal } \\
\text { ocurrencia irse del lugar } \\
\text { de trabajo, todos los días, } \\
\text { exactamente a la misma } \\
\text { hora permitida y estable- } \\
\text { cida como de salida". }\end{array}$} & 6 & $\begin{array}{l}\text { ya que no } \\
\text { es posible } \\
\text { asegurar que } \\
\text { no hayan } \\
\text { existido } \\
\text { motivaciones } \\
\text { subjetivas }\end{array}$ & $\begin{array}{l}\text { Proceso } \\
\text { relacionar }\end{array}$ & \multirow[t]{2}{*}{$\begin{array}{l}\text { Juicio } \\
\text { - sanción } \\
\text { social } \\
\text { - Integridad }\end{array}$} \\
\hline & 7 & $\begin{array}{l}\text { que pudieran } \\
\text { haber } \\
\text { enturbiado su } \\
\text { testimonio en } \\
\text { el sentido de } \\
\text { beneficiar a su } \\
\text { hijo }\end{array}$ & $\begin{array}{l}\text { Proceso } \\
\text { relacional } \\
\text { [metáfora] }\end{array}$ & \\
\hline & 8 & $\begin{array}{l}\text { lo que no es } \\
\text { objetivamente } \\
\text { posible ni } \\
\text { razonable } \\
\text { reprochar a } \\
\text { una madre } \\
\text { en las } \\
\text { circunstancias } \\
\text { en que } \\
\text { aquellas } \\
\text { pudieron } \\
\text { producirse }\end{array}$ & $\begin{array}{l}\text { Proceso } \\
\text { mental }\end{array}$ & $\begin{array}{l}\text { Juicio } \\
\text { - sanción } \\
\text { social } \\
\text { - integridad } \\
\text { (neutral) }\end{array}$ \\
\hline
\end{tabular}

Fuente: Elaboración propia. 


\subsection{Análisis de estereotipos}

Los jueces se interesan por el incumplimiento del "deber de la madre", porque sería un medio para descubrir la verdad ${ }^{6} \mathrm{y}$, a la vez, refuerza un precepto moral según el cual las (buenas) madres no pueden omitir realizar oportunamente toda gestión útil para evitar un daño a sus hijos. Ante la encrucijada, se opta por explicitar la función epistémica del deber (ella no es creíble), aun cuando dicha función es débil, ya que de hecho muchas madres no auxilian a sus hijos oportunamente. La función epistémica parece, entonces, apoyarse en un estereotipo acerca del comportamiento de las buenas madres.

En este punto es necesario introducir una precisión respecto de la noción de estereotipo (Arena, 2015). En efecto, no todos los estereotipos parecen funcionar del mismo modo. Por un lado, existen estereotipos (que llamaremos descriptivos), que persiguen ofrecer información acerca del mundo y su dirección de ajuste es estereotipo-mundo. Ello quiere decir que si el estereotipo no coincide con el mundo, es una razón para abandonar o modificar el estereotipo ${ }^{7}$. Así, los estereotipos descriptivos pueden ser evaluados sobre la base de su correspondencia con las propiedades efectivas del grupo al que se refieren. Por ejemplo, el estereotipo según el cual los italianos son católicos. Por otro lado, existen estereotipos (que llamaremos normativos) que poseen una dirección de ajuste mundo-estereotipo, lo cual quiere decir que la falta de coincidencia entre mundo y estereotipo es una razón para modificar el mundo y no (necesariamente) una razón para modificar el estereotipo. En el caso de los estereotipos normativos existe una relación de deber ser entre la propiedad adscripta a los miembros del grupo y el hecho de ser miembro del grupo. Así, en principio, no tiene sentido preguntarse si los estereotipos normativos describen (o no) correctamente un determinado grupo o categoría de personas. Por el contrario, son normas que consideran que una determinada categoría de personas debería desempeñar ciertas tareas o determinados roles

\footnotetext{
$6 \quad$ Es una función epistémica del testimonio: si ella permaneció callada, lo que más adelante pudiera decir a favor de su hijo no sería cierto.

Esta distinción está presente en la definición mixta de estereotipo de género de la Corte Interamericana: "El (...) estereotipo de género se refiere a una preconcepción de atributos o características poseídas o papeles que son o deberían ser ejecutados por hombres y mujeres respectivamente" Caso González y otras ("Campo algodonero") vs. México. Sentencia del 16/11/2009. Serie C N²05, párrafo 401, CIDH.
} 
sociales. Como, por ejemplo, el estereotipo según el cual las madres deben ser amas de casa.

¿De qué tipo es el estereotipo usado por los jueces? La respuesta a esta pregunta es importante, porque los criterios para evaluar el uso de cada tipo de estereotipo son diferentes. Los jueces, por lo general, usan estereotipos descriptivos durante la actividad probatoria. Sin embargo, en el caso que analizamos, la referencia a aquello que una madre debería hacer para evaluar la credibilidad de la testigo parece indicar que los jueces usan un estereotipo normativo acerca del rol de las madres. No podemos aquí avanzar demasiado en el análisis de estos estereotipos y de su aceptabilidad en el proceso probatorio, pero vale la pena indicar que para que tengan fuerza como elementos de valoración de la prueba es necesario que, en ausencia de otra información, la probabilidad de que se produzca la acción en correspondencia con la norma dependerá de que se trate de un estereotipo aceptado por la comunidad a la que pertenece el sujeto al cual se atribuye. De otro modo, si no se tratara de una norma social - para cuya existencia es necesaria la regularidad de comportamiento por parte del grupo relevante-, no habría mayores elementos para sostener la expectativa acerca del comportamiento del sujeto en cuestión. Es decir, los jueces deberían probar (en este caso dieron por probado, sin ofrecer elementos adicionales) que el estereotipo normativo usado para comprender el comportamiento de las madres es una norma social en la comunidad a la que pertenece la madre del acusado. De otro modo, el juicio probatorio se apoyaría en la expectativa de que la madre del acusado se conforme a una norma inexistente o externa a su grupo social. Así, si es cierto que en una determinada comunidad existe una norma social según la cual los miembros de la comunidad deben hacer " $p$ ", el juez puede recurrir a la existencia de esa norma para tener como probable el hecho de que un miembro determinado de esa comunidad ha hecho "p".

Si el estereotipo al que apelan los jueces no es una norma social dentro de la comunidad a la que pertenece la testigo (o si los jueces no hacen ningún esfuerzo por demostrarlo), entonces el enunciado deja de ser probatorio y se transforma en lisa y llana crítica ideológica a su comportamiento. 
En este sentido, otro aspecto importante, y ligado al anterior, es la nominalización presente en la cláusula "esta conducta no resulta lógica" que a partir de la AT puede entenderse como la evocación de un juicio negativo de sanción social dirigido contra la madre, por la carencia de adecuación social. En general, las cláusulas del primer párrafo hacen explícito (inscriben) dos juicios positivos de estima social (tenacidad y capacidad) de la madre y dos juicios negativos: uno de estima social por ausencia de normalidad y otro de sanción social por carencia de adecuación social. Aquí se destaca que el objetivo de la evaluación de las primeras ocho cláusulas es la madre, quien es referida de forma explícita mediante el pronombre "ella" o de forma anafórica, mientras que el objeto evaluado en la cláusula 9 -que es el juicio más duro- es "la conducta". Este "movimiento" de sustitución del objeto enjuiciado permite afirmar que el primer párrafo contiene un juicio de valor negativo referido a la persona de la madre, que no está inscrito o explícito, sino que es evocado por medio de un conjunto de indicios lingüísticos como el conector "sin embargo" y los verbos "debió" y "esperó". Todos los cuales, activan un juicio negativo en torno a la posibilidad fáctica de acción que la madre, en opinión del tribunal sí tuvo, pero no aprovechó estando obligada a hacerlo.

En el segundo párrafo, el único complejo clausal que nos interesa es "la única persona que lo sitúa (al acusado) en un lugar distinto es la madre pero su relato es poco creíble". Específicamente, la cláusula "su relato es poco creíble" suscita la pregunta: ¿Qué o quién carece de la cualidad de ser creíble? A primera vista, en virtud de los significados ideacionales involucrados, parece ser que el objeto enjuiciado es el relato de la madre. Sin embargo, esta cláusula evoca un juicio negativo de sanción social que recae sobre aquella.

Los significados implícitos permiten inferir que uno de los motivos que los jueces tuvieron en consideración para preferir la versión de los hechos afirmada por el Ministerio Público fue una distancia ideológica entre el comportamiento de la madre y el estándar que el tribunal, a priori, tuvo para ponderar su conducta. Si bien la crítica personal directa está velada, implícita o evocada -y es claro que el escritor del texto se cuida de no hacerla explícita-, la lectura permite apreciar que el escritor transita desde juicios positivos de la tenacidad y capacidad 
de la madre hasta juicios negativos referidos a la veracidad de ese relato, a través de una ampliación de la crítica en los niveles de la estima social y de la adecuación social.

Antes de terminar, revisaremos dos dimensiones del análisis de la AT, las que si bien no conforman el núcleo del análisis, permiten visualizar cómo la composición del texto es funcional a la justificación de la decisión de no creer en el testimonio de la madre. Primero, desde el sistema de graduación, el texto expresa una progresión y amplificación de los significados interpersonales de las valoraciones de estima social y valoración social. El texto amplifica paulatinamente la crítica y ese proceso es una evidencia de cómo, a fin de cuentas, la ausencia de credibilidad del testimonio de la madre se funda, en gran parte, en una crítica personal e ideológica a su desempeño. He ahí la más posible razón de la sustitución (en el texto) del objeto criticado. En segundo lugar, es necesario advertir que, usando el sistema de compromiso de la AT y el desarrollo de ese sistema que ha propuesto Oteíza (2009), en el primer párrafo analizado la voz del escritor del texto se enmarca en un perfil de "evaluador" o "intérprete", es decir, los recursos de persuasión del texto se enfocan explícitamente en juicios de comportamiento de un modo explicativo y donde la interpretación no es explícita. Al final de este párrafo, en cambio, la voz transita hacia el perfil de un evaluador adjudicador, lo que implica que hay una toma de posición más o menos explícita acerca de lo que es "correcto".

\section{Conclusiones}

Las sentencias judiciales constituyen un macrogénero que se compone, entre otros microgéneros, de una etapa de consolidación de los hechos y de otros para la valoración de la prueba. A través del análisis de estos segmentos hemos podido advertir que el texto instancia un estereotipo normativo en cuanto a la conducta de una de las partes. Los estereotipos - a pesar de su fragilidad epistémica y de su carga ideológica- favorecen la construcción de sentencias que propician el ocultamiento de la incertidumbre en la decisión de dar por probados ciertos hechos. Esto es interesante porque el sistema procesal penal tiene mecanismos para enfrentar dicha clase de problemas, sin necesidad de ocultar espacios de incertidumbre a través del estándar de prueba "más allá de toda duda 
razonable" ${ }^{\text {. }}$. Esto último, por cuanto la ley dice que debe sancionarse a quienes se han apoderado con violencia de bienes ajenos y no a quienes los jueces crean que lo han hecho.

El texto de la sentencia analizada no presenta la evaluación de la prueba como una construcción compuesta de generalizaciones probabilísticas, sino como una conclusión de un razonamiento silogístico que tiene valor universal. La falta de reconocimiento de la incertidumbre que atraviesa la escrituración de la tarea judicial de construcción de lo probado nos otorga también pistas interesantes acerca de la función de las sentencias, en cuanto a fijar episodios problemáticos de la vida de las personas en términos de que no queden abiertos (como ocurriría en la investigación histórica o periodística). Aquello sería epistémicamente más correcto, pero implicaría reconocer la falibilidad de los jueces en la labor de construir representaciones de lo que habría ocurrido en el mundo, lo que entra en tensión con una visión mecanicista de su labor. Por cierto, un análisis depurado desde el punto de vista lingüístico puede permitir develar estos presupuestos que dan cuenta del ocultamiento de la subjetividad y de las ideologías a las que adhieren los jueces. Aquello sería importante, porque favorecería el esclarecimiento de la función social desempeñada por los jueces, incluso si lo que se descubriera no fuera como para "poner el grito en el cielo".

\section{Referencias bibliográficas}

Agüero, C. y Zambrano, J. P. (2009). La narración en las sentencias penales. Universum (Talca), 24(2), 28-41. https://doi. org/10.4067/s0718-23762009000200003

Agüero, C. y Zambrano, J. P. (2010). Integración metodológica para el estudio del texto de las sentencias penales chilenas. Convergencia, 17(54), 69-91. Recuperado de http:// www.scielo.org.mx/scielo.php?script=sci_arttext\&pid $=$ S1405-14352010000300004

$8 \quad$ Este estándar de prueba está regulado en el artículo 340 del Código Procesal Penal chileno y constituye una forma de distribución de los errores en que podrían incurrir los jueces por lagunas de información o de deficiente calidad epistémica de la prueba disponible (Coloma, 2010). 
Agüero, C. (2014). ¿Conforman las sentencias penales un género discursivo? Estudios filológicos, 53, 7-26. https://doi. org/10.4067/s0071-17132014000100001

Arena, F. (2015). Normative stereotypes in legal decision-making. En A. Capone \& E. Poggi (Comps.), Perspectives on pragmatics and law. The pragmatics of legal practice (pp. 379-399). Springer Switzerland: Cham.

Azuelos-Atías, S. (2006). Legal causality and criminal intent in the legal discourse. International Journal for the Semiotics of Law, 19(2), 183-205. https://doi.org/10.1007/s11196-0069016-y

Azuelos-Atías, S. (2007). A pragmatic analysis of legal proofs of criminal intent. Amsterdam: John Benjamins Publishing.

Azuelos-Atías, S. (2010). Semantically cued contextual implicatures in legal texts. Journal of Pragmatics, 42(3), 728-743. https://doi. org/10.1016/j.pragma.2009.07.009

Bolivar, A. (2007). El análisis interaccional del discurso: Del texto a la dinámica social. En A. Bolívar (Comp.), Análisis del discurso. ¿Por qué y para qué? ( pp.247-277). Caracas: Editorial CEC, S.A., Los Libros de El Nacional y la Universidad Central de Venezuela.

Bolivar, A. (2012). La construcción dialógica de un macrogénero: la crisis diplomática, En Shiro, M, et al. [edits.] Los géneros discursivos desde múltiples perspectivas: teorías y análisis, Madrid: Iberoamericana Vervuert.

Campos, M. (2007). El lenguaje de las ciencias jurídicas: nuevos retos y nuevas visiones. En E. Alcaraz, J. Martínez y F. Yus (Coords.), Las lenguas profesionales y académicas (pp. 155166). Barcelona: Ariel.

Ciapuscio, G. (2005). La noción de género en la lingüística sistémico funcional y en la lingüística textual. Revista signos, 38(57), 31-48. https://doi.org/10.4067/s0718-09342005000100003

Coloma, R. (2010). El debate sobre los hechos en los procesos judiciales. ¿Qué inclina la balanza? En D. Accatino (Comp.), Formación y valoración de la prueba en el proceso penal (pp. 87-117). Santiago de Chile: Abeledo Perrot-Legal Publishing.

Ducrot, O. (1988). Polifonía y argumentación. Cali: Universidad del Valle. 
Ducrot, O. (1983). Opérateurs argumentatifs et visée argumentative. Cahiers de Linguistique Française, 5. Genève Université de genéve, Unité de Linguistique Française pp. 5-36.

Eggins, S. y Martin, J. (2003). El contexto como género: una perspectiva lingüística funciona. Revista Signos, 36(54), 185-205. https:// doi.org/10.4067/s0718-09342003005400005

Eggins, S. (2004), Introduction to systemic functional linguistics (2nd ed.). London, New York: Continuum.

Halliday, M.A.K. (1978). Language as Social Semiotic The Social Interpretation of Language and Meaning. London Edward Arnold.

Halliday, M.A.K. \& Hasan, R. (1985). Language, Context and Text: Aspects of Language in a Social-Semiotic Perspective. Deakin University Press, Geelong.

Hood, S. y Martin, J. (2005). Invocación de actitudes: el juego de la gradación de la valoración en el discurso. Revista Signos, 38(58), 195-220. https://doi.org/10.4067/s071809342005000200004

García, M. y Agüero, C. (2014). Bases para el estudio de la dinámica discursiva en la comunidad jurídica chilena. Revista de Derecho (Valdivia), 27(1), 59-79. https://doi.org/10.4067/s071809502014000100003

Ghio, E. y Fernández, M. (2008). Lingüistica sistémico funcional: aplicaciones a las lenguas españolas. Santa Fe: Ediciones UNL.

Gil, J. M. y García, A. (2010). Transitividad, modo y tema en español: Un primer análisis en términos de la gramática de Cardiff. Revista Signos, 43(72), 71-98. https://doi.org/10.4067/s071809342010000100004

López, A. (2006). Los ordenadores del discurso enumerativos en la sentencia judicial: ¿estrategia u obstáculo? Revista de Llengua $i$ Dret, 25, 61-88. Recuperado de http://revistes.eapc.gencat. cat/index.php/rld/article/viewFile/2499/2978

López, A. (2010a). El género profesional del informe jurídico. Recomendar e interpretar la ley 159. En L. Chierichetti y G. Garofalo (Eds.), Lengua y derecho: líneas de investigación interdisciplinaria (pp. 159-184. Berna: Peter Lang.

López, A. (2010b). Documentos profesionales con destinatarios no expertos: el empleo de los mecanismos referenciales en la sen- 
tencia del 11M. Revista Signos, 43(72), 99-123. https://doi. org/10.4067/s0718-09342010000100005

Martin, J. (1997). Register and genre: modelling social context in functional linguistics - narrative genres. En E. Pedro (Ed.), Proceedings of the First Lisbon International Meeting on Discourse Analysis (pp. 305-344). Lisbon: Colibri/APL.

Martin, J. (2000). Beyond exchange: Appraisal systems in English. En G. Thompson \& S. Hunston (Comps.), Evaluation in text: Authorial stance and the construction of discourse (pp. 144175). Oxford: Oxford University Press.

Martin, J. \& Rose, M. (2003). Working with discourse: Meaning beyond the clause. London, New York: Continuum.

Montolío, E. y López, A. (2008). La escritura en el quehacer judicial: estado de la cuestión y presentación de la propuesta aplicada en la escuela judicial de España. Revista Signos, 41(66), 3364. https://dx.doi.org/10.4067/S0718-09342008000100002

Moyano, E. (2007). Enseñanza de habilidades discursivas en español en contexto pre-universitario: una aproximación desde la LSF. Revista Signos, 40(65), 573-608. https://doi.org/10.4067/ s0718-09342007000300009

Nølke, H., Fløttum, K., \& Norén, C. (2004). ScaPoLine. La théorie scandinave de la polyphonie linguistique. Paris: Kimé.

Oteíza, T. (2006). El discurso pedagógico de la historia: un análisis lingüístico sobre la construcción ideológica de la historia de Chile (1970-2001) (Vol. 1). Santiago de Chile: Frasis.

Oteíza, T. (2009). Solidaridad ideológica en el discurso de la historia: tensión entre orientaciones monoglósicas y heteroglósicas. Revista Signos, 42(70), 219-244. https://doi.org/10.4067/ s0718-09342009000200004

Portolés, J. (2007). Marcadores del discurso. Barcelona: Ariel.

White, P. (2000). The appraisal website. Recuperado de http://www. grammatics.com/appraisal/

Zambrano, J. P. y Agüero, C. (2009a). Multiculturalidad y discrecionalidad judicial en una sentencia penal: análisis desde Joseph Raz. Revista de derecho (Valparaíso), 32, 327-343. https:// doi.org/10.4067/s0718-68512009000100009

Zambrano, J. P. y Agüero, C. (2009b). El 'cultural point view' en una sentencia penal. Comentario a la sentencia de la primera 
sala del Tribunal Oral en lo Penal de Temuco-Chile, 23 de Noviembre de 2005. Revista Frónesis, Revista de Filosofía Jurídica Social y Política (Zulia), 16, 385-392. Recuperado de https://biblat.unam.mx/hevila/FronesisMaracaibo/2009/ vol16/no2/10.pdf

Zambrano, J.P.(2015).Derecho, ideologíay discurso.Alpha(Osorno), 40, 71-80. https://doi.org/10.4067/s0718-22012015000100006 